\title{
Composite One- to Six-Scroll Hidden Attractors in a Memristor-Based Chaotic System and Their Circuit Implementation
}

\author{
Ying Li, ${ }^{1}$ Xiaozhu Xia, ${ }^{1}$ Yicheng Zeng $\mathbb{D}^{1},{ }^{1}$ and Qinghui Hong $\mathbb{D}^{2}$ \\ ${ }^{1}$ School of Physics and Optoelectronic Engineering, Xiangtan University, Xiangtan, Hunan 411105, China \\ ${ }^{2}$ College of Computer Science and Electronic Engineering, Hunan University, Changsha, Hunan 410000, China
}

Correspondence should be addressed to Yicheng Zeng; yichengz@xtu.edu.cn and Qinghui Hong; 734798457@qq.com

Received 26 May 2020; Revised 13 August 2020; Accepted 8 October 2020; Published 20 November 2020

Academic Editor: Viorel-Puiu Paun

Copyright (C) 2020 Ying Li et al. This is an open access article distributed under the Creative Commons Attribution License, which permits unrestricted use, distribution, and reproduction in any medium, provided the original work is properly cited.

Chaotic systems with hidden multiscroll attractors have received much attention in recent years. However, most parts of hidden multiscroll attractors previously reported were repeated by the same type of attractor, and the composite of different types of attractors appeared rarely. In this paper, a memristor-based chaotic system, which can generate composite attractors with one up to six scrolls, is proposed. These composite attractors have different forms, similar to the Chua's double scroll and jerk double scroll. Through theoretical analysis, we find that the new system has no fixed point; that is to say, all of the composite multiscroll attractors are hidden attractors. Additionally, some complicated dynamic behaviors including various hidden coexisting attractors, extreme multistability, and transient transition are explored. Moreover, hardware circuit using discrete components is implemented, and its experimental results supported the numerical simulations results.

\section{Introduction}

Recently, much effort has been devoted to the analysis of various chaotic systems owing to its potential applications [1-4]. To our knowledge, chaotic attractors are categorized as either self-excited attractors [5-7] or hidden attractors [8-11]. It should be particularly pointed out that hidden attractors have neither homoclinic nor heteroclinic orbits; thus, the Shil'nikov theorem [12] cannot be utilized to verify the existence of chaos, so that hidden chaotic systems attract great attention of scholars, and increasing number of researchers begin to study the rich dynamic behaviors of them.

As is known to all, multiscroll chaotic systems have aroused extensive interests for their much complicated dynamical properties than single-scroll ones. So, it is a more desirable task to explore the hidden multiscroll chaotic system, and there have been emerging related literatures on this topic. In 2016, Jafari et al. presented a novel no-equilibrium chaotic system with multiscroll hidden chaotic sea by introducing a sine function into a 3D chaotic system [13]. In the same year, Hu et al. constructed two simple 3D chaotic systems without equilibrium based on an improved Sprott A system by adding a nonlinear function, from which hidden multiscroll attractors can be obtained [14]. Later, a new class of PWL dynamical system without equilibrium whose hidden chaotic attractors can display grid multiscroll has been introduced [15]. Deng and Wang proposed a multiscroll hidden chaotic system that has only two stable nodefoci equilibrium points [16]. Hong et al. investigated a novel method for designing multidirection multibutterfly chaotic attractors (MDMBCA) without reconstructing nonlinear functions [17]. Furthermore, some memristive chaotic systems, which can generate hidden multiscroll or multiwing chaotic attractors, are reported $[18,19]$. However, all of the hidden multiscroll attractors discussed above are of the same type attractor repeated in unidirectional or multidirection ones. Are there hidden multiscroll attractors, which are composed of different attractors? We are surprised that the answer is positive. 
If an attractor is compounded from two or more different single-scroll attractors, which is called composite attractor, its dynamic characteristics are more complicated. The composite attractor has been already discovered in some self-excited chaotic systems. For example, Cang et al. proposed a Lorenzlike system with composite structure by controlling system parameters [20]. Zhang et al. reported an autonomous-system-based approach for creating composite chaotic attractors from a class of generalized Lorenz systems via switching control [21]. And then, Xiong et al. constructed a chaotic system, which can generate composite four-scroll attractor by adding a symbolic function into a 3D jerk system [22]. However, although the complexity of dynamic behavior of these chaotic systems, which can produce composite attractors, has increased, the operation methods and state equations have also been correspondingly complicated, and the circuit experiment is difficult to realize. Besides, these composite multiscroll chaotic attractors mentioned above are limited to self-excited attractors, and the hidden composite multiscroll attractors have not been reported up to now. It is obvious that designing a simple chaotic system, which can generate hidden composite multiscroll attractors, is a meaningful task.

Based on these considerations, we introduce a quadratic flux-controlled memristor into the $3 \mathrm{D}$ jerk chaotic system to construct a memristor-based hidden composite multiscroll chaotic system, which can produce composite one- to sixscroll hidden attractors through changing only one system parameter. It is pointed out that the composite six-scroll hidden attractor is composed of a Chua's double-scroll attractor and two jerk double-scroll attractors. Compared with [22], this new memristor-based hyperchaotic system has more complex dynamic behaviors including hyperchaotic composite multiscroll hidden attractors and extreme multistability phenomenon. These composite multiscroll attractors break the previous pattern of the same singlescroll attractor that appeared repeatedly and has not been reported in previous literature.

The rest of this paper is organized as follows. In Section 2 , the mathematical model of the proposed memristive hyperchaotic system and its typical attractor are presented. In Section 3, complex hidden dynamic behaviors of this new system including controllable multiscroll hidden chaotic or hyperchaotic attractors, coexistence of hidden attractors, extreme multistability, and transient transition behavior are described. In Section 4, the corresponding circuit implementation and experiment results are presented. The conclusion is presented in the last section.

\section{Memristive Hyperchaotic System and Its Typical Attractors}

In 2016, Kengne et al. performed a simple 3D autonomous jerk system with cubic nonlinearity [23], which is described as

$$
\left\{\begin{array}{l}
\dot{x}=y, \\
\dot{y}=a z, \\
\dot{z}=x-b y-z-x^{3}
\end{array}\right.
$$

where $x, y, z$ are state variables, and $a, b$ are positive tunable parameters. In order to construct a memristor-based hyperchaotic system, a quadratic flux-controlled memristor model [24] described in equation (2) is added into system (1):

$$
\left\{\begin{array}{l}
\dot{u}=v, \\
i=(m+n u) v
\end{array}\right.
$$

where $u, v$ and $i$ denote the internal state of the memristor, the input of the memristor, and the output of the memristor model, respectively. The $m, n$ are two real parameters of the memristor model. So, we constructed a 4D memristor-based chaotic system, which can generate composite multiscroll hidden attractors, and it is described as

$$
\left\{\begin{array}{l}
\dot{x}=y+z, \\
\dot{y}=a z-\operatorname{sgn}(z)+p, \\
\dot{z}=2 x-b y-z-k W(u) y-x^{3}, \\
\dot{u}=c y
\end{array}\right.
$$

in which $x, y, z$ are state variables, $a, b, c, k$ are real parameters, $W(u)=(m+n u)$ is the memductance function, and $p$ is positive control parameter. From a dissipative perspective, the divergence of the new system (3) can easily be obtained as follows:

$$
\nabla V=\frac{\partial \dot{x}}{\partial x}+\frac{\partial \dot{y}}{\partial y}+\frac{\partial \dot{z}}{\partial z}+\frac{\partial \dot{u}}{\partial u}=-1,
$$

at any given point $(x, y, z, u)$ of the state space. Consequently, the general condition of dissipativity related to the existence of attractive sets in our model is satisfied. The equilibrium points of system (3) can be obtained by solving the following equations: $\dot{x}=\dot{y}=\dot{z}=\dot{u}=0$. It is obvious that the equations have no real solutions when parameter $p$ is positive. That is to say, system (3) has no equilibrium, and it belongs to hidden chaotic system.

When fixing the control parameters $a=3.5, b=0.8$, $m=0.1, n=0.3, p=0.01, k=0.2, c=0.01$ and selecting the initial conditions as $(0.1,0.1,0.1,0.1)$, a composite six-scroll hidden chaotic attractor and its Poincaré map on $y=0$ section as well as power spectrum are displayed in Figures 1(a)-1(c). As is depicted in Figure 1(a), the six-scroll attractor is composed of a Chua's double-scroll attractor and two jerk double-scroll attractors, and the two small jerk double-scroll attractors are inside the large Chua's doublescroll attractor, respectively. It is surprising that the composite six-scroll hidden chaotic attractors have never appeared before as far as we know.

\section{Dynamic Analysis and Numerical Investigations}

In this section, multifarious complicated dynamic behaviors including controllable multiscroll hidden attractors, coexisting of hidden chaotic or hyperchaotic attractors, extreme multistability, and transient transition behaviors of proposed system (3) are investigated in detail by means of 


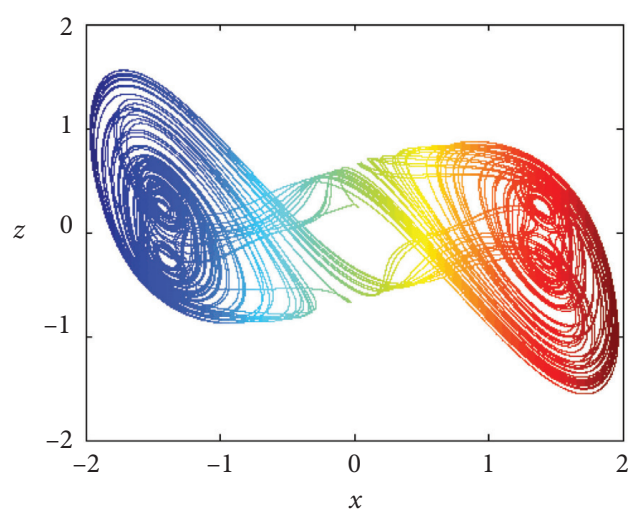

(a)

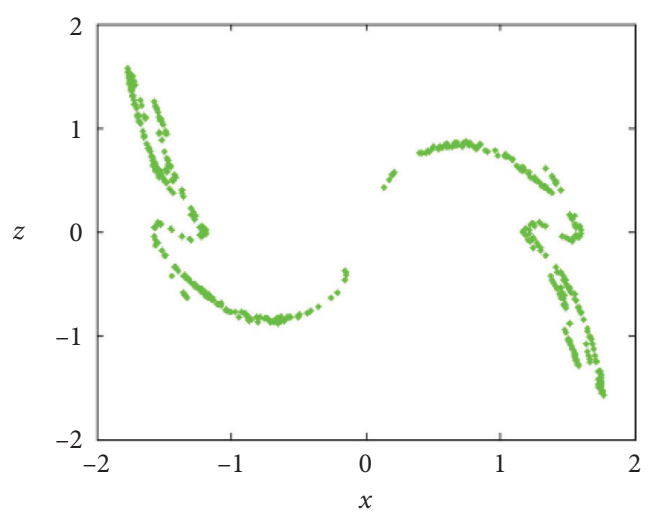

(b)

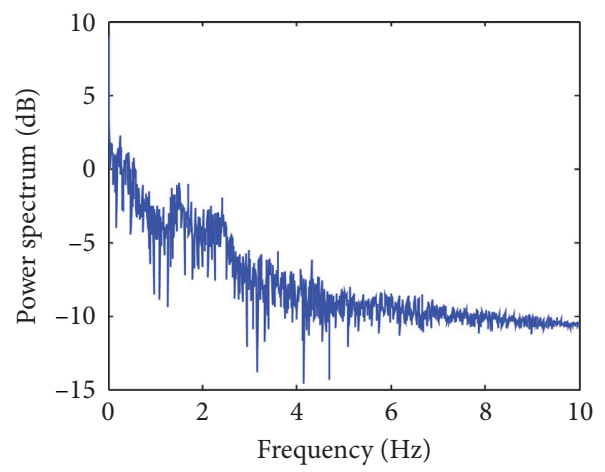

(c)

Figure 1: (a) The composite six-scroll hidden chaotic attractor. (b) The corresponding Poincare mapping on $y=0$ section. (c) The corresponding power spectrum.

Lyapunov exponent spectra, bifurcation diagrams, phase portraits, and time series. Note that all numerical simulations are carried out on MATLAB software, and the first 20000 points are discarded in the phase portraits.

\subsection{Controllable Multiscroll Hidden Chaotic or Hyperchaotic} Attractors. One interesting phenomenon is that the newly proposed system (3) can generate composite one- to sixscroll hidden attractors by changing only one system parameter. Fixing the control parameters $b=0.8, m=0.1$, $n=0.3, k=0.2, c=0.01, p=0.01$ and changing the parameter $\mathrm{a}$ in the interval of $[1.3,5]$ with the initial conditions $(0.1$, $0.1,0.1,0.1)$, the corresponding bifurcation diagram is drawn in Figure 2(a). Additionally, using the famous Wolf method [25], the corresponding Lyapunov exponent spectrum proves the complexity of system (3) as depicted in Figure 2(b).

In reference to Figure 2(b), system (3) undergoes chaotic and hyperchaotic routes as follows: chaotic $(1.3 \leq a<1.5) \longrightarrow$ hyperchaotic $\quad(1.5 \leq a<2.65) \longrightarrow$ chaotic $(2.56 \leq a<3.06) \longrightarrow$ hyperchaotic $(3.06 \leq a<3.42) \longrightarrow$ chaotic $(3.42 \leq a<5)$. What surprised us is that system (3) generates composite one- to six-scrolls- hidden attractors when selecting appropriate parameters, and these attractors may exhibit either a symmetric or asymmetric structure. It is rare to see such attractors in an asymmetric system. Some typical hidden chaotic or hyperchaotic attractors with different values of parameter $a$ are shown in Figures 3(a)3(1). Note that Figures 3(a) and 3(b) display a pair of asymmetric single-scroll hidden chaotic attractors at $a=1.38$ and 2.92. Figures $3(\mathrm{c})$ and $3(\mathrm{~d})$ show a pair of symmetric double-scroll hidden hyperchaotic attractors at $a=2.3$ and 2.55 , and the corresponding Lyapunov exponents are $(0.213$, $0.179,0,-1.381)$ and $(0.255,0.209,0,-1.444)$, respectively. Figures 3(e) and 3(f) exhibit two different double-scroll hidden chaotic attractors at $a=2.946$ and 5. And a pair of asymmetric composite 3 -scroll hidden chaotic and hyperchaotic attractors are drawn in Figures $3(\mathrm{~g})$ and $3(\mathrm{~h})$ with $a=2.974$ and 3.117. Furthermore, a pair of symmetric composite 4-scroll hidden chaotic and hyperchaotic attractors, a composite 5-scroll hidden chaotic attractor, and a composite 6-scroll hidden hyperchaotic attractor are presented in Figures 3(i) and 3(l) with $a=3.03$, 3.076, 3.055, and 3.125 , respectively. To explain more intuitively and clearly, the different types of attractors with different values of parameter $a$ are listed in Table 1. Consequently, the unusual controllable multiscroll hidden chaotic or hyperchaotic attractors of system (3) are innovative.

3.2. Coexistence of Hidden Attractors. Nowadays, attractor coexisting is one of the common nonlinear dynamic phenomena, which is a chaotic system performing more than one concurrent attractor for a given set of system parameters under the different initial conditions [26-32]. In this section, 


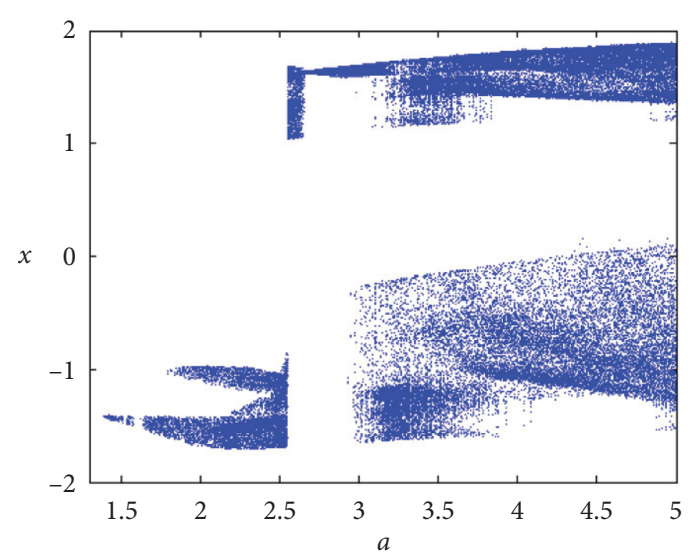

(a)

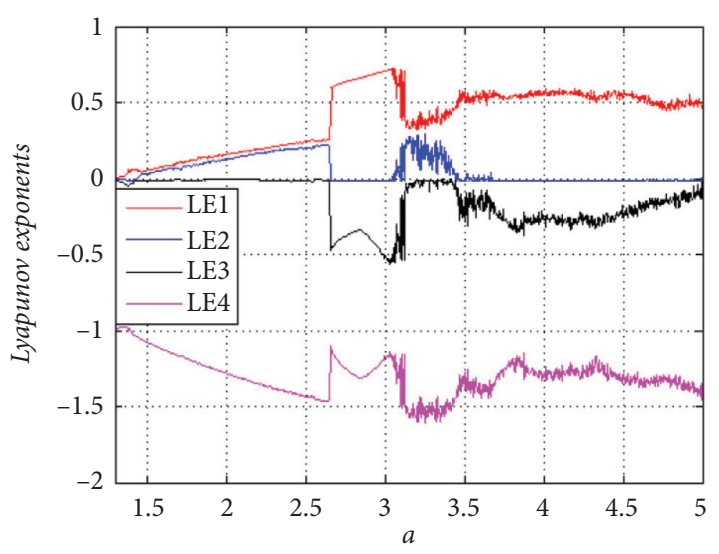

(b)

Figure 2: Dynamic behavior with increasing the parameter a in the interval of $[1.3,5]$. (a) Bifurcation diagrams of $x$. (b) Lyapunov exponent spectrum.

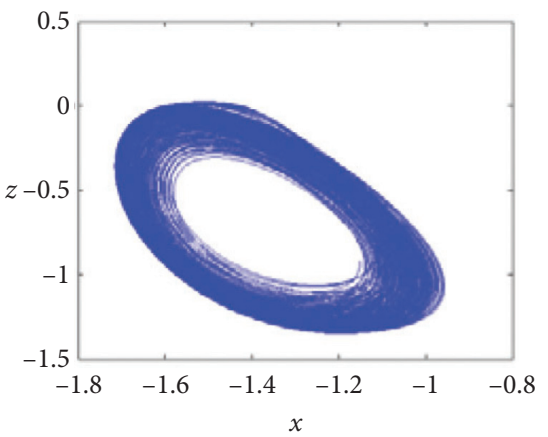

(a)

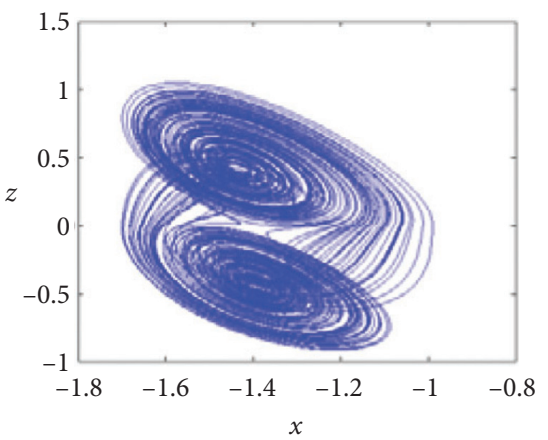

(d)

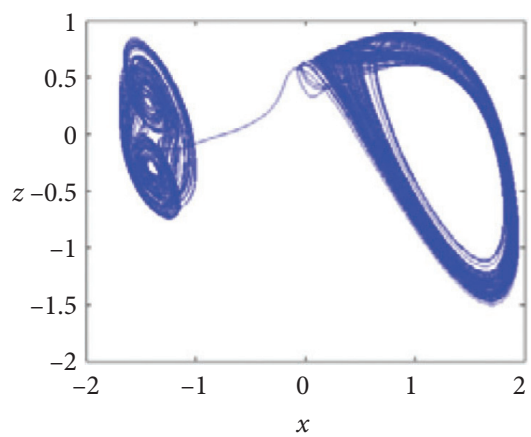

(g)

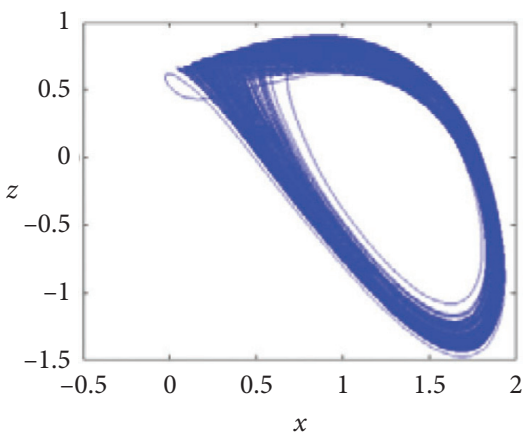

(b)

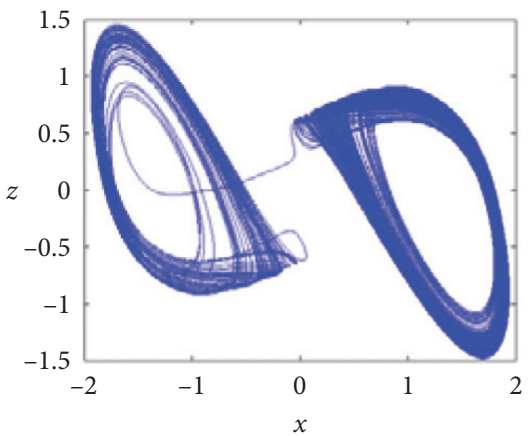

(e)

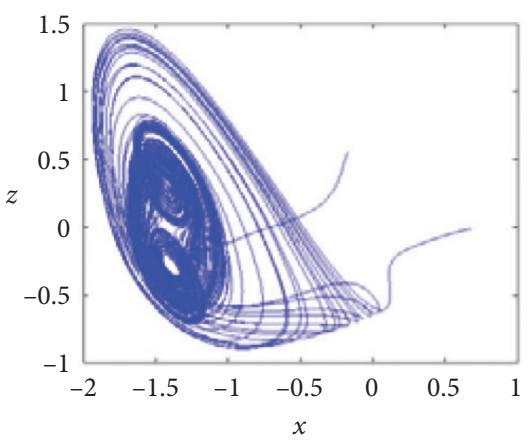

(h)

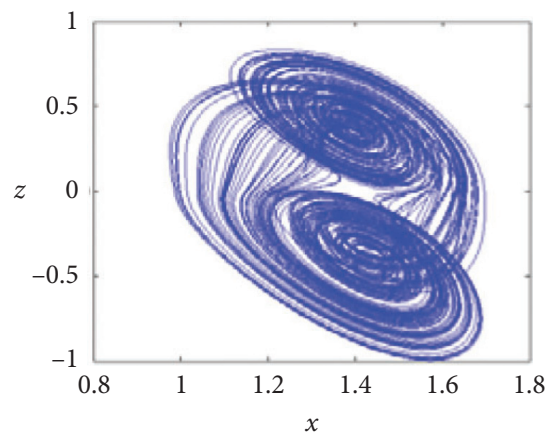

(c)

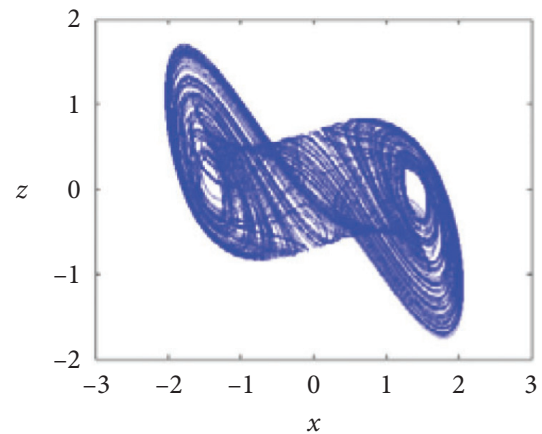

(f)

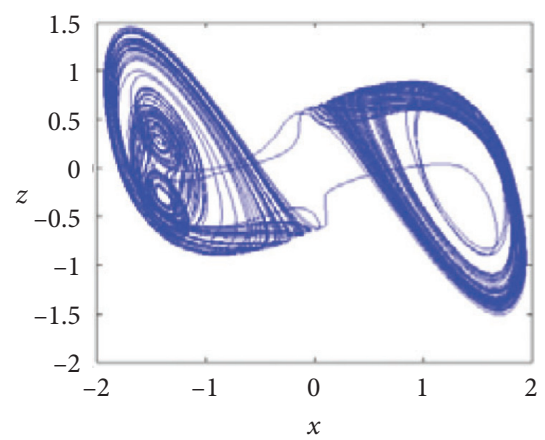

(i)

Figure 3: Continued. 


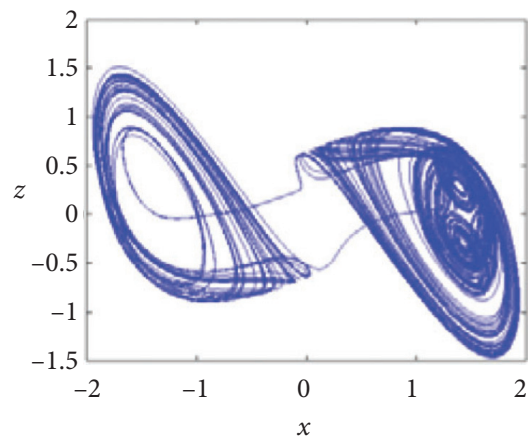

(j)

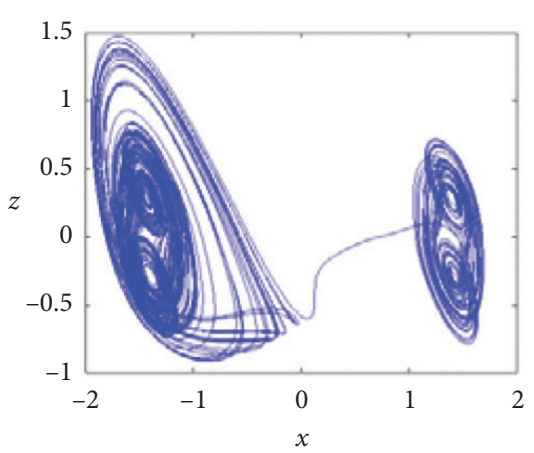

$(\mathrm{k})$

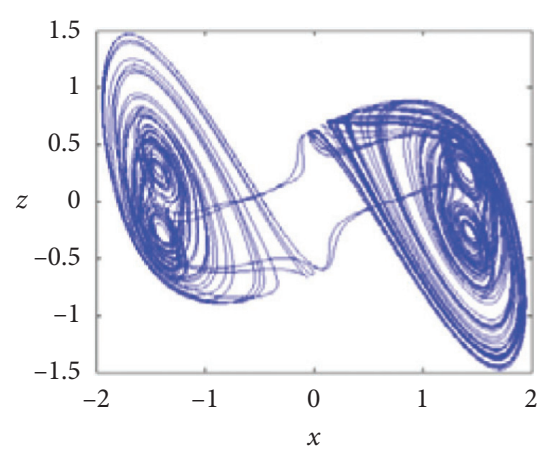

(1)

Figure 3: Some typical phase portraits with different parameter $a$. (a, b) A pair of asymmetric single-scroll chaotic attractors. (b) A pair of asymmetric single-scroll chaotic attractors. (c, d) A pair of symmetric double-scroll chaotic attractors. (e, f) Two different double-scroll chaotic attractors. (g, h) Two different composite 3-scroll chaotic attractors. (i, j) A pair of symmetric composite 4-scroll chaotic and hyperchaotic attractors. (k) A composite 5-scroll chaotic attractor. (l) A composite 6-scroll hyperchaotic attractor.

TABLE 1: Composite one- to six-scroll attractors of system (3) with variation of parameter $a$.

\begin{tabular}{lcccc}
\hline Parameter $a$ & $\mathrm{LE}_{S}$ & Chaotic or hyperchaotic & The number of scrolls of hidden attractor & Diagrams \\
\hline 1.38 & $(+, 0,-,-)$ & Chaotic & Single-scroll & Figure 3(a) \\
2.92 & $(+, 0,-,-)$ & Chaotic & Single-scroll & Figure 3(b) \\
2.3 & $(+,+, 0,-)$ & Hyperchaotic & Double-scroll & Figure 3(c) \\
2.55 & $(+,+, 0,-)$ & Hyperchaotic & Double-scroll & Figure 3(d) \\
2.946 & $(+, 0,-,-)$ & Chaotic & Double-scroll & Figure 3(e) \\
5 & $(+, 0,-,-)$ & Chaotic & 3-scroll & Figure 3(f) \\
2.974 & $(+, 0,-,-)$ & Chaotic & 3 -scroll & Figure 3(g) \\
3.117 & $(+,+, 0,-)$ & Hyperchaotic & 4 -scroll & Figure 3(h) \\
3.03 & $(+, 0,-,-)$ & Chaotic & 4-scroll & Figure 3(i) \\
3.076 & $(+,+, 0,-)$ & Hyperchaotic & 5-scroll & Figure 3(j) \\
3.055 & $(+, 0,-,-)$ & Chaotic & 6-scroll & Figure 3(k) \\
3.125 & $(+,+, 0,-)$ & Hyperchaotic & & Figure 3(l) \\
\hline
\end{tabular}

the coexistence of hidden attractors with some specific parameters setting and initial conditions in system (3) is discussed. By selecting the control parameters as $b=0.8$, $m=0.1, n=0.3, k=0.2, c=0.01, p=0.01$, the coexisting bifurcation diagram of the state variable $x$ with respect to the parameter $a$ is described in Figure 4(a). Figure 4(b) displays the Lyapunov exponent spectrum under the initial conditions $(-0.1,-0.1,-0.1,-0.1)$, which is in accordance with the Lyapunov exponent spectrum under the initial conditions $(0.1,0.1,0.1,0.1)$ in Figure 2(b). Various composite multiscroll chaotic or hyperchaotic hidden coexisting attractors are shown in Figure 5; they are including symmetric attractors and asymmetric attractors coexisting, odd number of attractors, and even number of attractors coexisting. The more detailed information is displayed in Table 2. All in all, it is informative and rare that so many composite multiscroll attractors coexist, which injects new blood into the field of chaos coexisting.

3.3. Extreme Multistability Relying on Memristor Initial Condition. In this subsection, we further studied the extreme multistability phenomenon [33-38] in system (3). To reveal extreme multistability phenomenon relying on memristor initial condition, the typical parameters $a=3.05$, $b=0.8, c=0.01, m=0.1, n=0.3, k=0.2, p=0.01$ and initial conditions $x(0)=0.1, y(0)=0.1, z(0)=0.1$ are fixed. When the memristor initial condition $u(0)$ is increased gradually from -4.8 to 10 , the bifurcation diagram of the state variable $x$ and the Lyapunov exponent spectrum are plotted in Figures 6(a) and 6(b), respectively. As can be seen from Figure 6, the system has extremely rich dynamic characteristics.

To explain the coexistence of infinite number of attractors in system (2) more intuitively, several special values of $u$ are selected as shown in Table 3 and the phase diagrams of the infinite coexisting attractors as shown in Figure 7.

Specially, when setting $a=20, b=0.8, m=0.1, n=0.3$, $k=0.2, c=0.01, p=0.01$ and initial conditions $x(0)=0.1, y$ $(0)=0.1, z(0)=0.1$, and change the memristor initial condition $u(0)$ in the region of $[0,8]$, the bifurcation diagram is plotted in Figure 8. In light of Figure 8, it is clear that the system goes through reverse period doubling bifurcation route from chaos to periodic. In order to illustrate this phenomenon of extreme multistability, there are seven types of hidden attractors, which are presented in Figure 9. In fact, more different types of hidden attractors can be obtained 


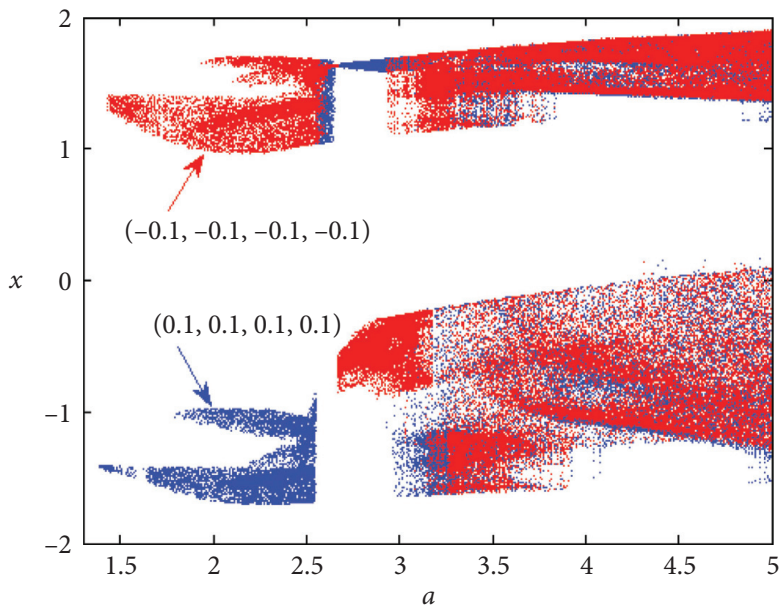

(a)

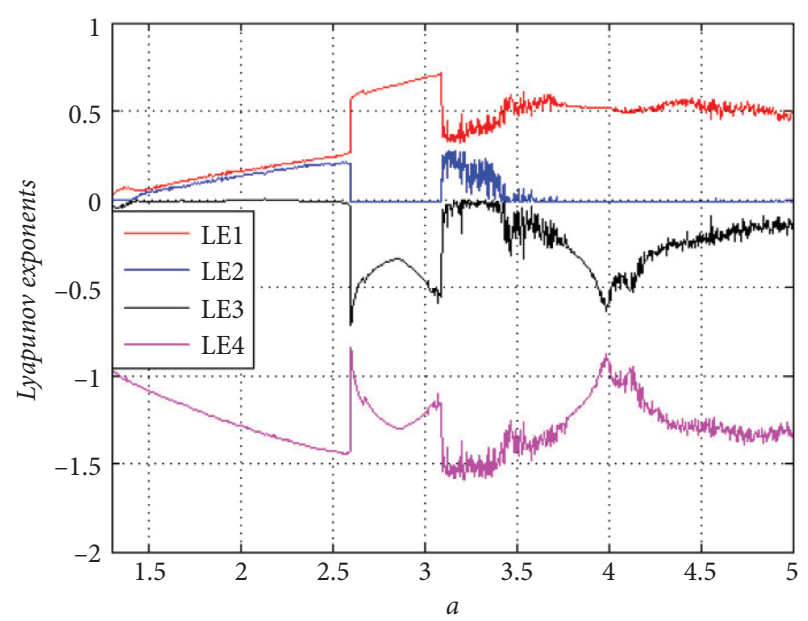

(b)

FIGURE 4: Coexisting bifurcation behaviors with increasing the parameter a in the interval of [1.3, 5]. (a) Coexisting bifurcation diagrams of the state variable $(x)$. (b) Lyapunov exponent spectrum with initial conditions $(-0.1,-0.1,-0.1,-0.1)$.

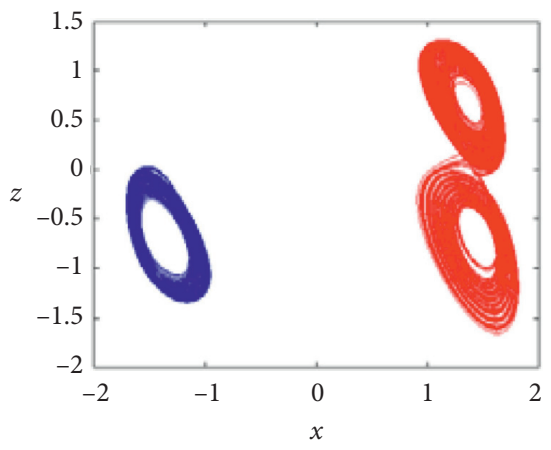

(a)

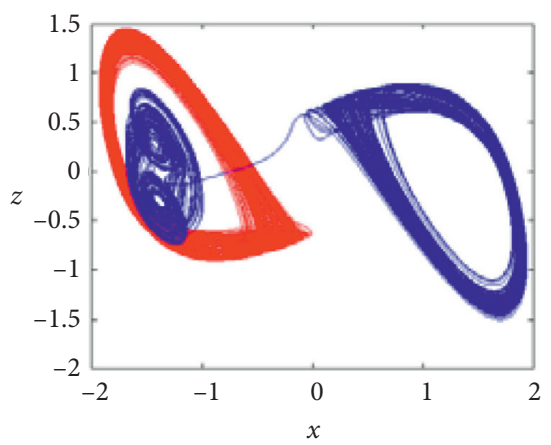

(d)

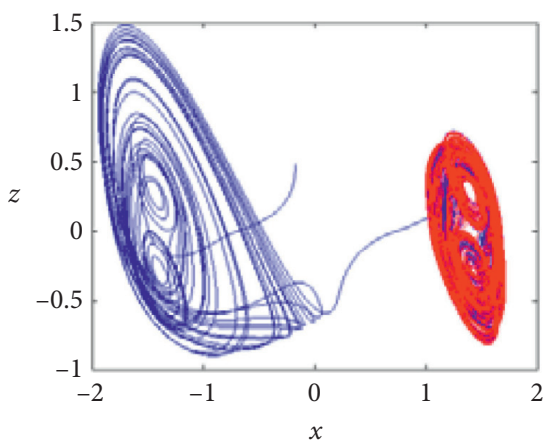

(g)

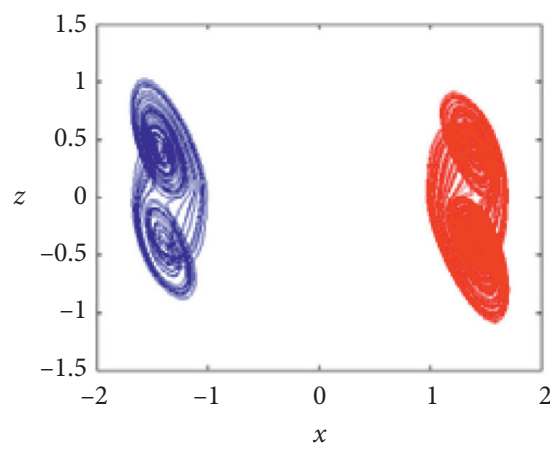

(b)

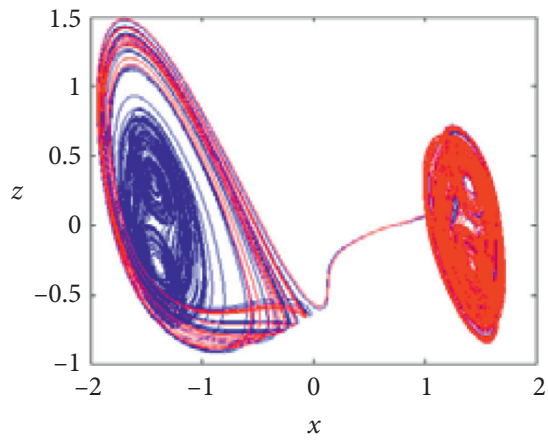

(e)

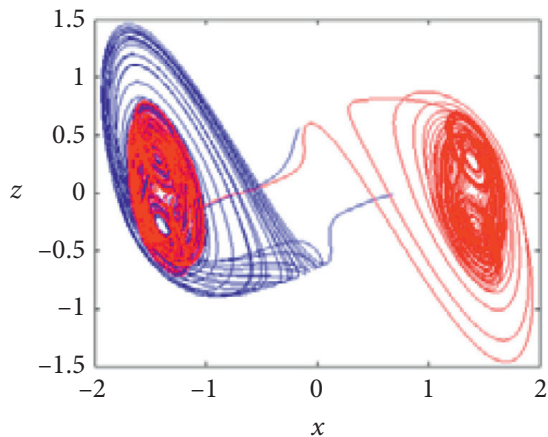

(h)

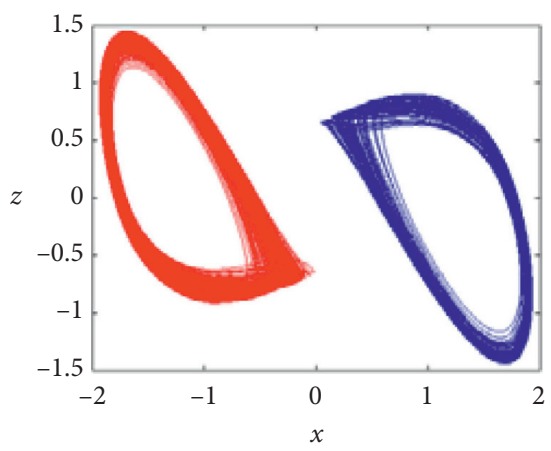

(c)

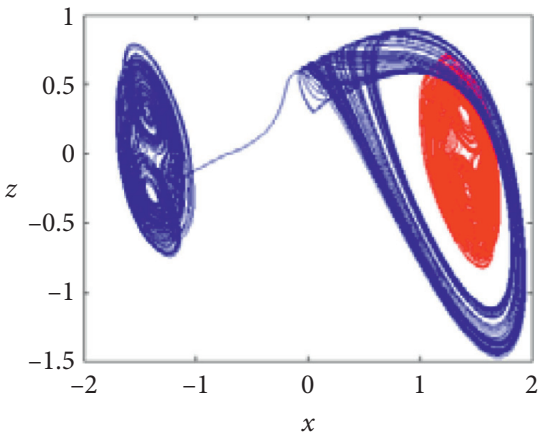

(f)

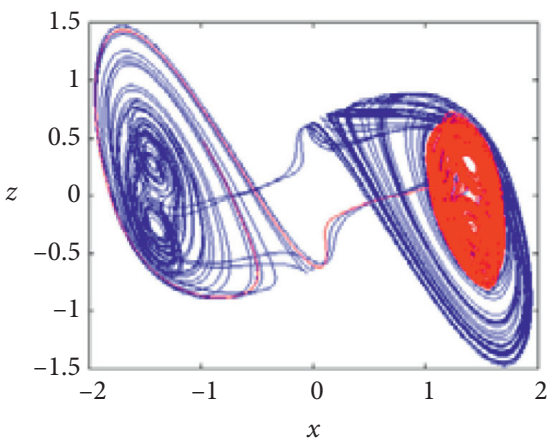

(i)

FIgURE 5: The coexisting chaotic or hyperchaotic hidden attractors with different values of parameter $a$. 
TABLE 2: The coexisting multiscroll attractors of system (3) with different values of parameter $a$.

\begin{tabular}{lccc}
\hline Parameter $a$ & Chaotic or hyperchaotic & Coexisting attractors & Diagrams \\
\hline 1.38 & Chaotic & A single scroll and a double-scroll & Figure 5(a) \\
2.3 & Hyperchaotic & Two symmetric double-scroll & Figure 5(b) \\
2.92 & Chaotic & Two symmetric single-scroll & Figure 5(c) \\
2.974 & Chaotic & A 3-scroll and a single-scroll & Figure 5(d) \\
3.055 & Chaotic & A 5-scroll and a 3-scroll & Figure 5(e) \\
3.095 & Hyperchaotic & A 3-scroll and a double-scroll & Figure 5(f) \\
3.105 & Hyperchaotic & A 5-scroll and a double-scroll & Figure 5(g) \\
3.117 & Hyperchaotic & A 3-scroll and a 4-scroll & Figure 5(h) \\
3.125 & Hyperchaotic & A 6-scroll and a double-scroll & Figure 5(i) \\
\hline
\end{tabular}

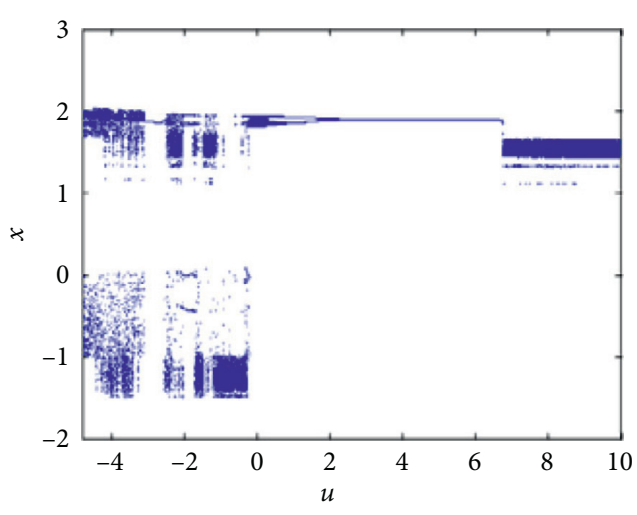

(a)

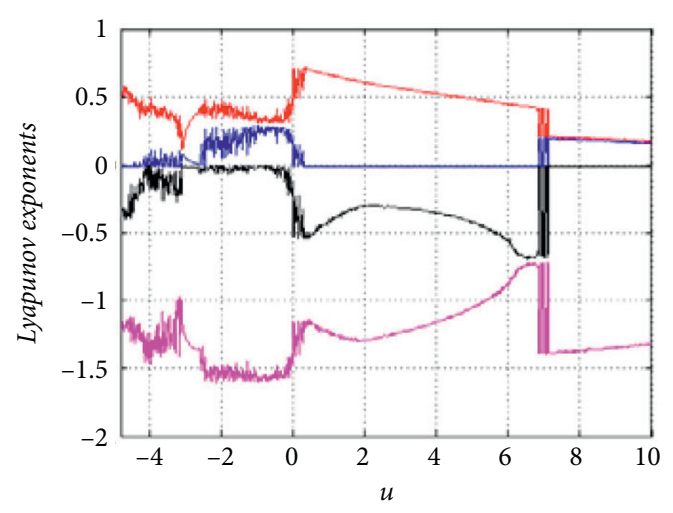

(b)

FIgURE 6: Bifurcation diagram and the Lyapunov exponent spectrum with respect to the initial conditions $u(0)$ in region of $[-4.8,10]$.

TABLE 3: Extreme multistability behaviors of system (3).

\begin{tabular}{lcc}
\hline Initial conditions & $\begin{array}{c}\text { Types of coexisting multiple } \\
\text { attractors }\end{array}$ & Diagrams \\
\hline$(0.1,0.1,0.1,-1.76) ;(0.1,0.1,0.1,0.8) ;(0.1,0.1,0.1,0.14) ;(0.1,0.1$, & Two single scroll and two double- & $\begin{array}{c}\text { Figure } 7(\mathrm{a}) \text { red, green, cyan, } \\
\text { scroll }\end{array}$ \\
$0.1,0.59)$ & Three double-scroll & Figure $7(\mathrm{~b})$ magenta, green, cyan. \\
$(0.1,0.1,0.1,-4.3) ;(0.1,0.1,0.1,0.4) ;(0.1,0.1,0.1,7.2)$ & Two 3-scroll & Figure 7(c) magenta, green. \\
$(0.1,0.1,0.1,0.23) ;(0.1,0.1,0.1,0.37)$ & Two 4-scroll & Figure 7(d) magenta, green. \\
$(0.1,0.1,0.1,-0.18) ;(0.1,0.1,0.1,-0.1)$ & Two 5-scroll & Figure 7(e) magenta, green. \\
$(0.1,0.1,0.1,-1.29) ;(0.1,0.1,0.1,-0.42)$ & Two 6-scroll & Figure 7(f) magenta, green. \\
$(0.1,0.1,0.1,-3.4) ;(0.1,0.1,0.1,-1.8)$ & &
\end{tabular}

under the different initial conditions. In general, Figures 7-9 intuitively reveal the coexisting infinitely many hidden hyperchaotic attractors, which indicates that system (3) possesses hidden extreme multistability indeed.

3.4. Transient Transition Behavior. In system exhibiting transient transition, the orbit is from one state (chaotic, periodic) to another state (chaotic, periodic) for a finite time interval before settling into a final state. Transient chaos with boundary crisis is often encountered in nonlinear dynamic systems [22, 39-43]. For the sake of verifying transient transition behavior in system (3), the system parameters are considered as $a=3.115, \quad b=0.8, \quad m=0.1, \quad n=0.3, k=0.2$, $c=0.01, p=0.01$, while the initial conditions are selected as $(0.1,0.1,0.1,0.1)$ and the time is $t=1000 \mathrm{~s}$, and a phase diagram of six scrolls and the corresponding time-domain waveform with state variables $x$ and $z$ are shown in Figures 10(a)-10(c). Figure 11 displays three different phase diagrams with different simulation time and the Lyapunov exponent spectrum with increased time. From the Lyapunov exponent spectrum in Figure 11(d), it is clearly seen that there are two Lyapunov exponents greater than zero at $t=80 \mathrm{~s}$; that is to say, system (3) transitions from chaotic to hyperchaotic in the time of $t=80 \mathrm{~s}$.

\section{Circuit Implementation and Experiment Results}

Circuit implementation provides an alternative approach to explore the novel no-equilibrium memristive hyperchaotic system (3), and it is the key to the chaos application. In this part, a suitable electrical circuit is designed and a hardware 


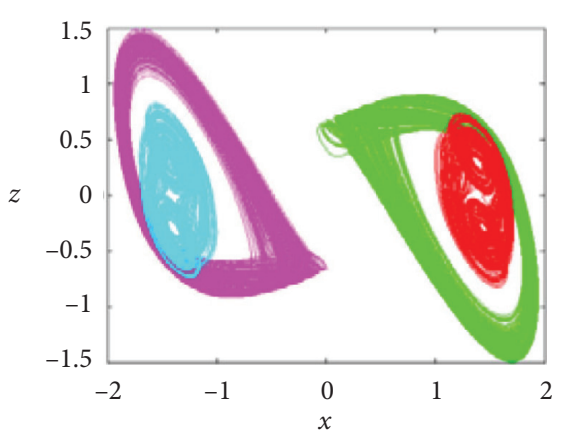

(a)

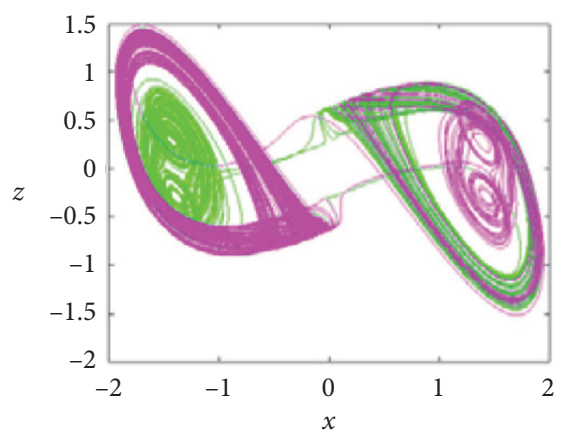

(d)

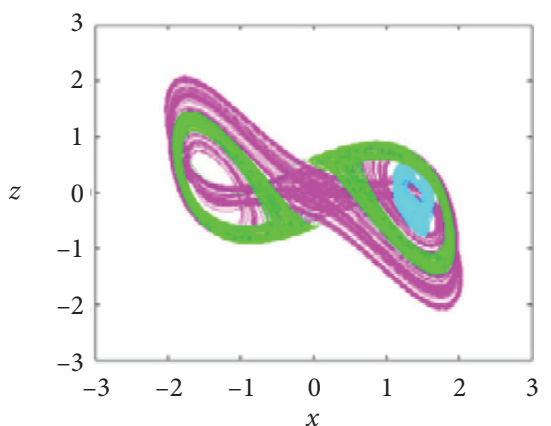

(b)

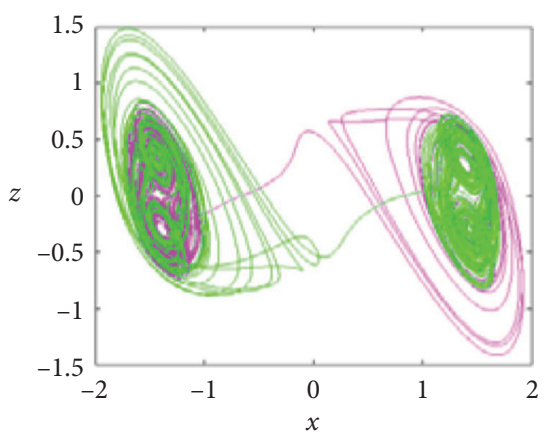

(e)

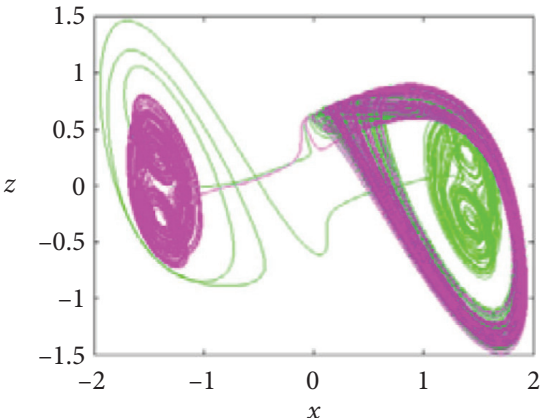

(c)

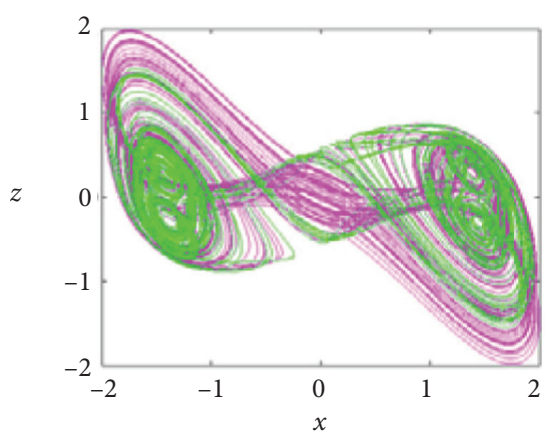

(f)

FIgURE 7: The coexistence of infinite number of attractors on $x-z$ plane with different initial condition $u(0)$.

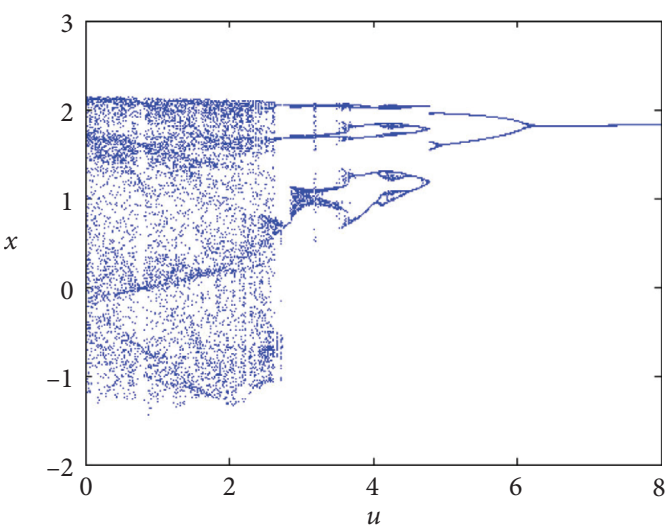

FIGURE 8: Bifurcation diagram with respect to the initial conditions $u(0)$ in region of $[0,8]$.

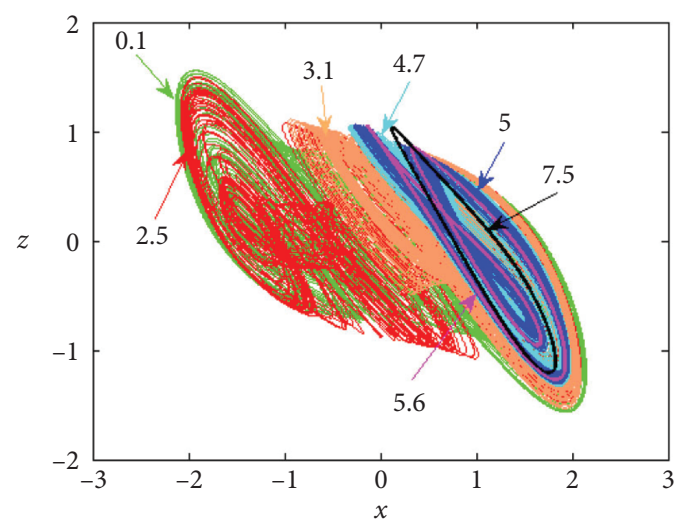

FIgURE 9: The coexistence of infinite number of attractors on $x-z$ plane with different initial condition $u(0)$, the different attractors corresponding to the different values of $u(0)$ are marked with different colors. 


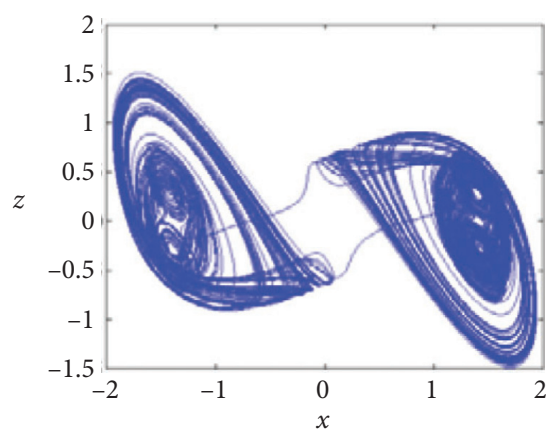

(a)

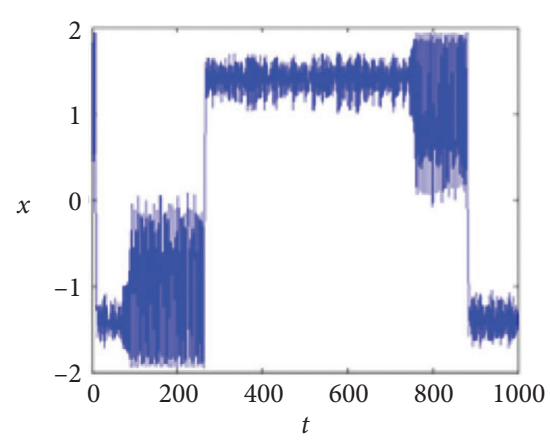

(b)

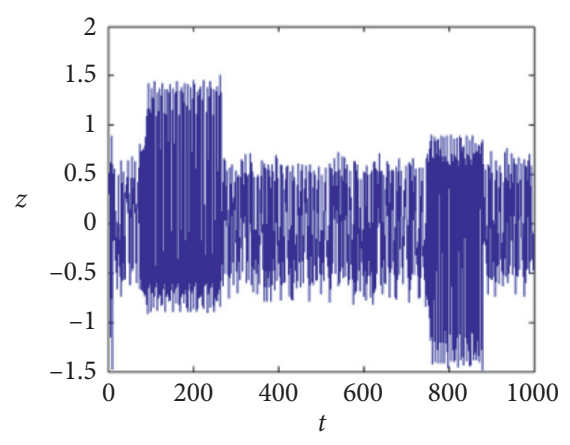

(c)

Figure 10: The transient transition behaviors of system (3). (a) A phase diagram of six-scroll hidden chaotic attractors. (b) Time-domain waveform of the variable $(x)$. (c) Time-domain waveform of the variable $z$.

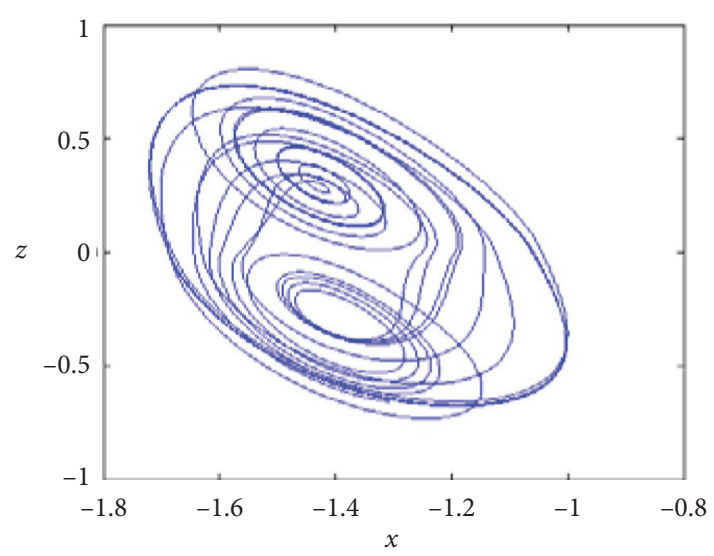

(a)

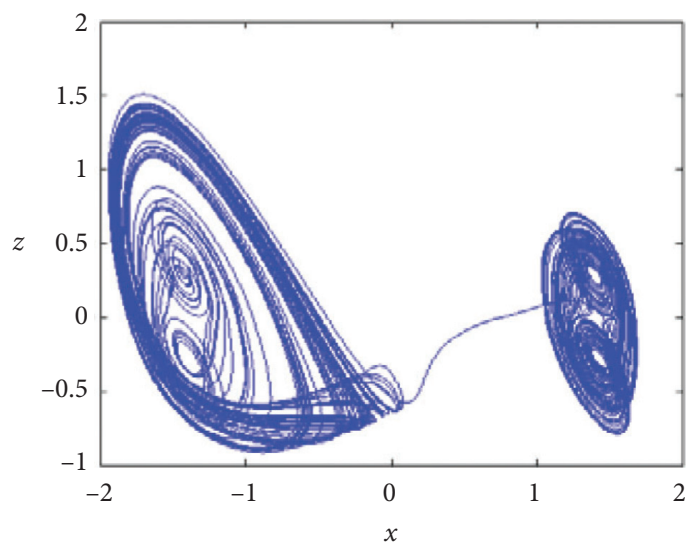

(c)

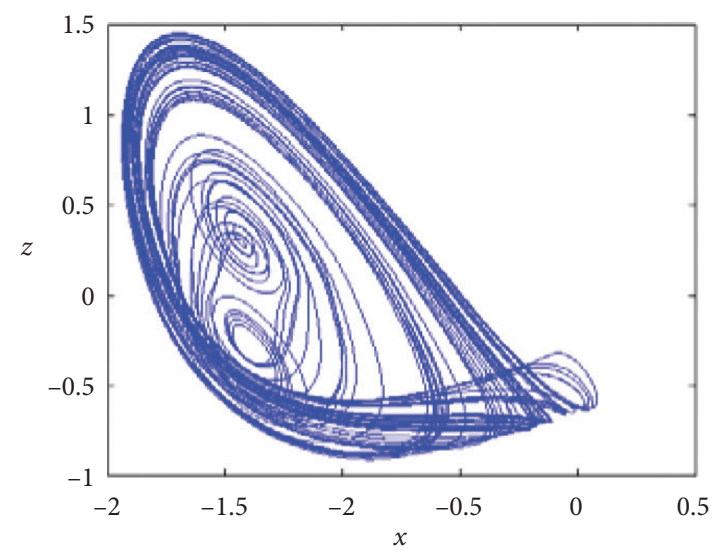

(b)

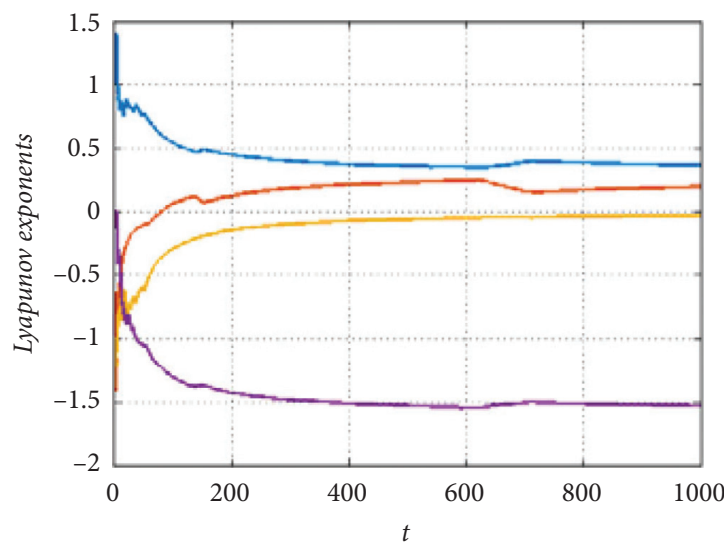

(d)

FIgURE 11: The transient transition behaviors with system (3). (a) A phase diagram of double-scroll hidden hyperchaotic attractors with $t=80 \mathrm{~s}$. (b) A phase diagram of 3-scroll hidden chaotic attractors with $t=250 \mathrm{~s}$. (c) A phase diagram of 5-scroll hidden hyperchaotic attractors with $t=750 \mathrm{~s}$. (d) The Lyapunov exponent spectrum with increased time in the region of $[0,1000]$.

circuit is implemented to verify the theoretical results obtained previously. The circuit diagram of the proposed system (3) is provided in Figure 12, and the circuits in the dashed box are constructed for the symbolic function circuit and memristor equivalent circuit.
The operational amplifiers of Figure 12 are LF353, whose supply voltages are $\pm 15 \mathrm{~V}$. And multipliers $A_{1}, A_{2}, A_{3}$ are AD633 with output coefficient of 0.1. Based on system (3), select the system parameters as $a=3.5, b=0.8, m=0.1$, $n=0.3, k=0.2, c=0.01, p=0.01$ and let $\gamma=\gamma_{0} \mathrm{t}$, where 

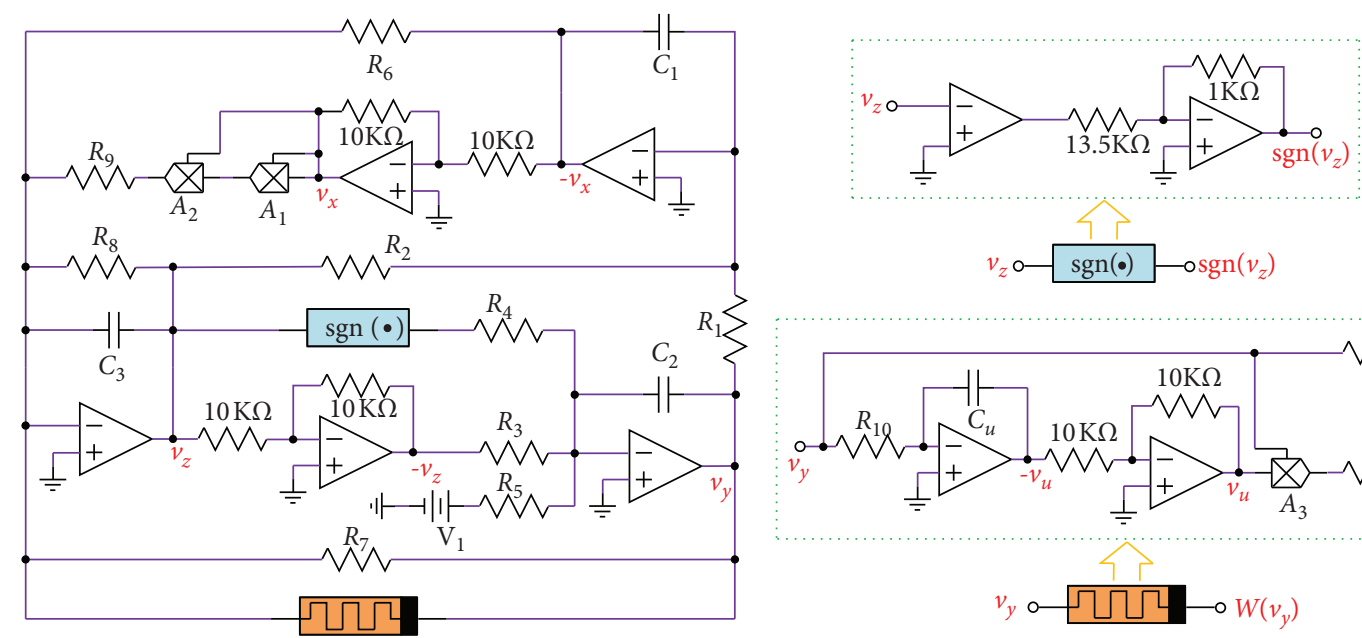

$v_{z} \circ \operatorname{sgn}\left(\bullet-\circ \operatorname{osgn}\left(v_{z}\right)\right.$
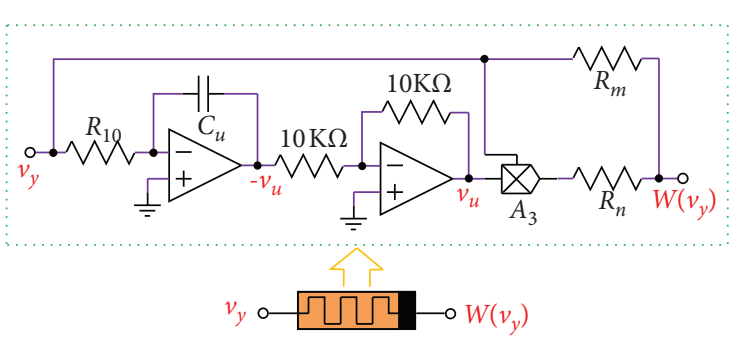

FIgURE 12: Circuit implementation of system (3).

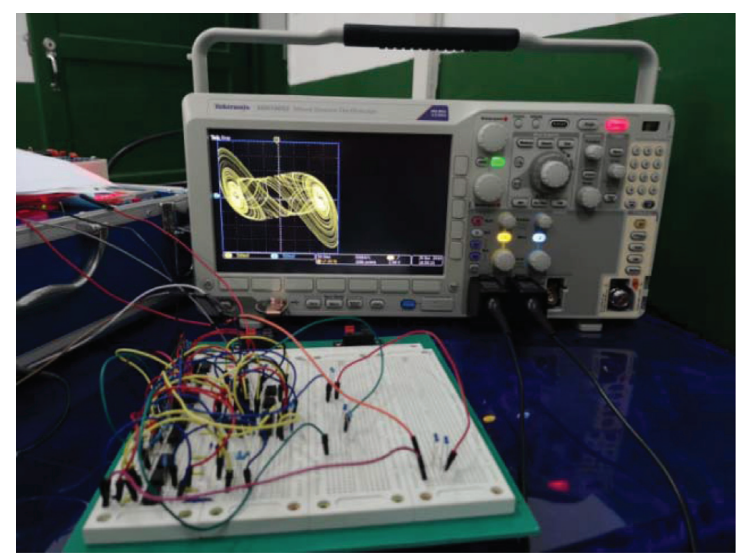

Figure 13: The hardware circuit test setup to realize system (3).

$\gamma_{0}=10000$ is the time-scale transformation factor and system

(3) can be rewritten by

$$
\left\{\begin{array}{l}
\dot{x}=10000 y+10000 z \\
\dot{y}=35000 z-10000 \operatorname{sign}(z)+100 \\
\dot{z}=20000 x-8000 y-10000 z-2000 W(u) y-10000 x^{3} \\
\dot{v}=100 y
\end{array}\right.
$$

where $W(u)=(m+n u)$. Considering Kirchhoff's law, the circuit equation of system (3) can be written as

$$
\left\{\begin{array}{l}
\frac{\mathrm{d} v_{x}}{\mathrm{~d} t}=\left(\frac{1}{R_{1} C_{1}}\right) v_{y}-\left(\frac{1}{R_{2} C_{1}}\right) v_{z}, \\
\frac{\mathrm{d} v_{y}}{\mathrm{~d} t}=\left(\frac{1}{R_{3} C_{2}}\right) v_{y}-\left(\frac{1}{R_{4} C_{2}}\right) \operatorname{sign}\left(v_{z}\right)+\left(\frac{1}{R_{5} C_{2}}\right) V_{1}, \\
\frac{\mathrm{d} v_{z}}{\mathrm{~d} t}=\left(\frac{1}{R_{6} C_{3}}\right) v_{x}-\left(\frac{1}{R_{7} C_{3}}\right) v_{y}-\left(\frac{1}{R_{8} C_{3}}\right) v_{z}-\left(\frac{1}{R_{m} C_{3}}\right) v_{y}-\left(\frac{1}{10 R_{n} C_{3}}\right) v_{u} v_{y}-\left(\frac{1}{100 R_{9} C_{3}}\right) v_{x}^{3}, \\
\frac{\mathrm{d} v_{u}}{\mathrm{~d} t}=\left(\frac{1}{R_{10} C_{u}}\right) v_{y} .
\end{array}\right.
$$




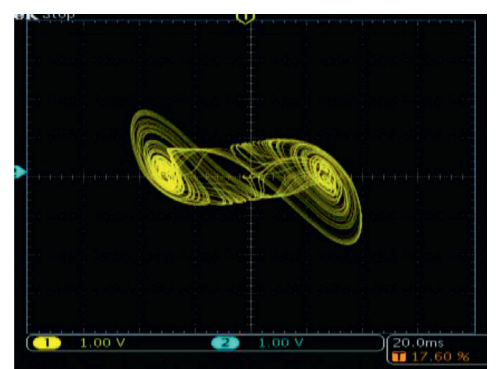

(a)

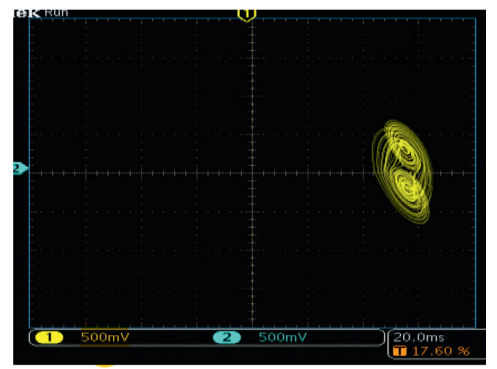

(d)

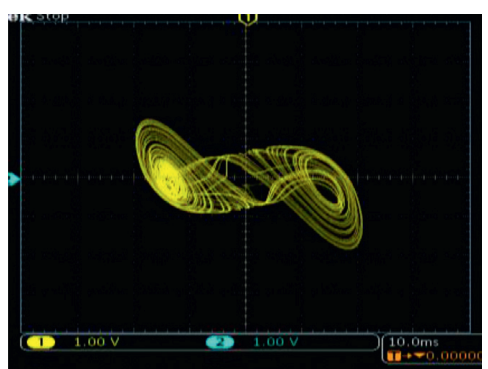

(b)

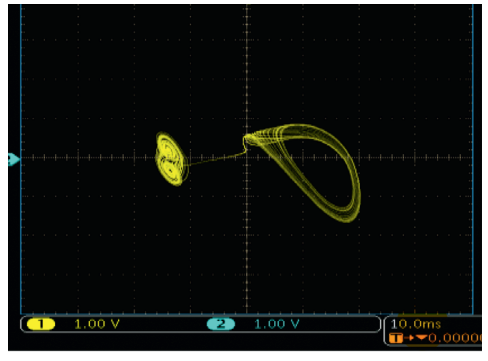

(e)

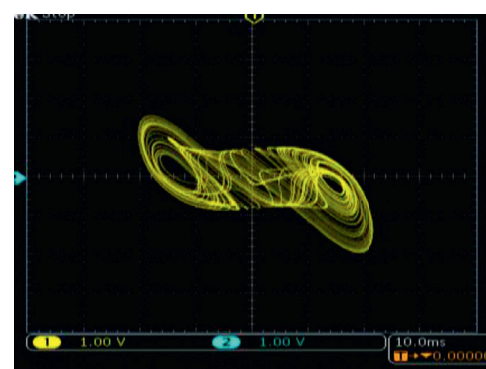

(c)

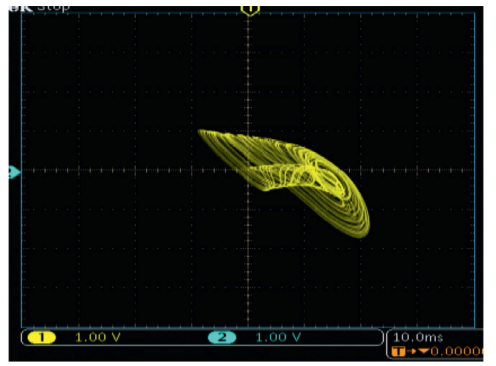

(f)

FIGURE 14: The screenshots of the digital oscilloscope of the different composite multiscroll attractors with different $R_{3}$. (a) Composite 6scroll with $R_{3}=2.86 \mathrm{k} \Omega$; (b) composite 4 -scroll with $R_{3}=3.3 \mathrm{k} \Omega$; (c) double-scroll with $R_{3}=0.5 \mathrm{k} \Omega$; (d) double-scroll with $R_{3}=4.348 \mathrm{k} \Omega$; (e) composite 3-scroll with $R_{3}=3.362 \mathrm{k} \Omega$; (f) single-scroll with $R_{3}=0.263 \mathrm{k} \Omega$.

Here, $v_{x}, v_{y}, v_{z}$ and $v_{u}$ are the voltages on capacitors $C_{1}, C_{2}$, $C_{3}$ and $C_{u}$, respectively, and the capacitors $C_{1}=C_{2}=C_{3}=C_{u}=10 \mathrm{nF}, V_{1}=-1 \mathrm{~V}$. Comparing equation (5) with equation (6) and keeping the corresponding coefficients equal, one gets $R_{1}=R_{2}=R_{4}=R_{8}=10 \mathrm{k} \Omega$, $R_{3}=2.86 \mathrm{k} \Omega, \quad R_{5}=R_{10}=10 \mathrm{M} \Omega, \quad R_{6}=5 \mathrm{k} \Omega, \quad R_{7}=12.5 \mathrm{k} \Omega$, $R_{9}=100 \Omega, R_{m}=500 \mathrm{k} \Omega, R_{n}=16.7 \mathrm{k} \Omega$. Then, according to the circuit diagram in Figure 12, some off-the-shelf discrete components are used on the breadboard to build the hardware circuit as shown in Figure 13 and the parameters in the circuit are set as above. We can see a composite sixscroll chaotic attractor as shown in Figure 14(a) by using digital oscilloscope, which is well consistent with the phase diagram simulated with MATLAB in Figure 1(a). Lastly, keeping the values of $R_{1}, R_{2}, R_{4}, R_{5}, R_{6}, R_{7}, R_{8}, R_{9}, R_{m}$ and $R_{n}$ unchanged, and adjusting the values of $R_{3}$ to appropriate resistance, other composite multiscroll attractors can be obtained from this hardware circuit as shown in Figures 14(b)-14(f), which also agree with the results of MATLAB simulation aforementioned. All of what is discussed above demonstrates the effectiveness and feasibility of system (3).

\section{Conclusion}

In this paper, a memristor-based chaotic system with abundant dynamic behaviors is reported. Compared with other memristor-based chaotic systems, the new system can display composite one- to six-scroll hidden attractors by changing only one system parameter. And the composite multiscroll attractors are composed of different types of attractors. For example, a composite six-scroll attractor is composed of a
Chua's double-scroll attractor and two jerk double-scroll attractors. The coexistence of symmetric and asymmetric attractors with different numbers of scrolls also reflects the particularity of the system. Furthermore, extreme multistability phenomenon and chaotic transient transition are investigated in the system. Finally, the feasibility of the proposed chaotic system is verified by hardware circuit, and the results match very well with the numerical simulations. Due to the fact that such a new chaotic system constructed in this paper has complex dynamic properties, it could be utilized advantageously in chaos-based engineering applications including image encryption, random bit generation, and chaosbased secure communication, and for future investigation.

\section{Data Availability}

The MATLAB simulation program data used to support the findings of this study are included within the supplementary information file. The simulation data of the study are reflected in the manuscript.

\section{Conflicts of Interest}

The authors declare that they have no conflicts of interest.

\section{Acknowledgments}

The work was supported by the National Natural Science Foundations of China under Grant no. 62071411 and the Fundamental Research Funds for the Central Universities under Grant no. 531118010418. 


\section{Supplementary Materials}

The following is the supplementary description for the given supplementary material. Appendix A: MATLAB simulation code for phase portrait. Appendix B: MATLAB simulation code for bifurcation diagram. Appendix C: MATLAB simulation code for Lyapunov exponent spectrum. (Supplementary Materials)

\section{References}

[1] F. C. Moon and P. D. Stiefel, "Coexisting chaotic and periodic dynamics in clock escapements," Philosophical Transactions of the Royal Society A: Mathematical, Physical and Engineering Sciences, vol. 364, no. 1846, pp. 2539-2564, 2006.

[2] E. F. D. Goufo and I. T. Toudjeu, "Analysis of recent fractional evolution equations and applications," Chaos, Solitons \& Fractals, vol. 126, pp. 337-350, 2019.

[3] C. Han, "An image encryption algorithm based on modified logistic chaotic map," Optik, vol. 181, pp. 779-785, 2019.

[4] C. Chen, L. Li, H. Peng, and Y. Yang, "Fixed-time synchronization of memristor-based BAM neural networks with time-varying discrete delay," Neural Networks, vol. 96, pp. 47-54, 2017.

[5] G. Litak, M. Borowiec, A. Syta, and K. Szabelski, "Transition to chaos in the self-excited system with a cubic double well potential and parametric forcing," Chaos, Solitons \& Fractals, vol. 40, no. 5, pp. 2414-2429, 2009.

[6] J. C. Sprott, "Strange attractors with various equilibrium types," The European Physical Journal Special Topics, vol. 224, no. 8, pp. 1409-1419, 2015.

[7] E. F. Doungmo Goufo, "Mathematical analysis of peculiar behavior by chaotic, fractional and strange multiwing attractors," International Journal of Bifurcation and Chaos, vol. 28, no. 10, Article ID 1850125, 2018.

[8] Z. Wei, I. Moroz, J. C. Sprott et al., "Hidden hyperchaos and electronic circuit application in a 5D self-exciting homopolar disc dynamo," Chaos: An Interdisciplinary Journal of Nonlinear Science, vol. 27, no. 3, Article ID 33101, 2017.

[9] E. F. D. Goufo, "On chaotic models with hidden attractors in fractional calculus above power law," Chaos, Solitons \& Fractals, vol. 127, pp. 24-30, 2019.

[10] M. A. Jafari, E. Mliki, A. Akgul et al., "Chameleon: the most hidden chaotic flow," Nonlinear Dynamics, vol. 88, no. 3, pp. 2303-2317, 2017.

[11] S. Jafari, J. C. Sprott, and S. M. R. Golpayegani, "Elementary quadratic chaotic flows with no equilibria," Physics Letters A, vol. 377, no. 9, pp. 699-702, 2013.

[12] L. P. Hashemi Golpayegani, "A case of the existence of a countable number of periodic motions," Soviet Mathematics Docklady, vol. 6, pp. 163-166, 1965.

[13] S. Jafari, V. T. Pham, and T. Kapitaniak, "Multiscroll chaotic sea obtained from a simple 3D system without equilibrium," International Journal of Bifurcation and Chaos, vol. 26, no. 2, Article ID 1650031, 2016.

[14] X. Hu, C. Liu, L. Liu, J. Ni, and S. Li, "Multi-scroll hidden attractors in improved Sprott A system," Nonlinear Dynamics, vol. 86, no. 3, pp. 1725-1734, 2016.

[15] R. J. Escalante-Gonzalez and E. Campos-Canton, "A class of Piecewise Linear Systems without equilibria with 3-D grid multiscroll chaotic attractors," Institute of Electrical and Electronics Engineering Transactions on Circuits and Systems II: Express Briefs, vol. 66, no. 8, pp. 1456-1460, 2018.
[16] Q. Deng and C. Wang, "Multi-scroll hidden attractors with two stable equilibrium points," Chaos: An Interdisciplinary Journal of Nonlinear Science, vol. 29, no. 9, Article ID 93112, 2019.

[17] Q. Hong, Y. Li, X. Wang et al., “A versatile pulse control method to generate arbitrary multi-direction multi-butterfly chaotic attractors," Institute of Electrical and Electronics Engineering Transactions on Computer-Aided Design of Integrated Circuits and Systems, vol. 38, no. 8, pp. 1480-1492, 2018.

[18] X. Hu, C. Liu, L. Liu et al., "Multi-scroll hidden attractors and multi-wing hidden attractors in a 5-dimensional memristive system," Chinese Physics B, vol. 26, no. 11, Article ID 110502, 2017.

[19] Z. Ling, C. Wang, and L. Zhou, "A novel no-equilibrium hyperchaotic multi-wing system via introducing memristor," International Journal of Circuit Theory \& Applications, vol. 46, no. 2, 2017

[20] S. Cang, Z. Wang, Z. Chen, and H. Jia, "Analytical and numerical investigation of a new lorenz-like chaotic attractor with compound structures," Nonlinear Dynamics, vol. 75, no. 4, pp. 745-760, 2014.

[21] C. Zhang, S. Yu, and G. Chen, "Design and implementation of compound chaotic attractors," International Journal of Bifurcation and Chaos, vol. 22, no. 5, Article ID 1250120, 2012.

[22] L. Xiong, S. Zhang, Y. Zeng, and B. Liu, "Dynamics of a new composite four-Scroll chaotic system," Chinese Journal of Physics, vol. 56, no. 5, pp. 2381-2394, 2018.

[23] J. Kengne, Z. T. Njitacke, and H. B. Fotsin, "Dynamical analysis of a simple autonomous jerk system with multiple attractors," Nonlinear Dynamics, vol. 83, no. 1-2, pp. 751-765, 2016.

[24] B. C. Bao, X. Zou, Z. Liu et al., "Generalized memory element and chaotic memory system," International Journal of Bifurcation and Chaos, vol. 23, no. 8, 2013.

[25] A. Wolf, J. B. Swift, H. L. Swinney, and J. A. Vastano, "Determining Lyapunov exponents from a time series," Physica D: Nonlinear Phenomena, vol. 16, no. 3, pp. 285-317, 1985.

[26] W. Zhou, G. Wang, Y. Shen et al., "Hidden coexisting attractors in a chaotic system without equilibrium point," International Journal of Bifurcation and Chaos, vol. 28, no. 10, 2018.

[27] V. Varshney, S. Sabarathinam, A. Prasad et al., "Infinite number of hidden attractors in memristor-based autonomous duffing oscillator," International Journal of Bifurcation and Chaos, vol. 28, no. 1, Article ID 1850013, 2018.

[28] D. Mathale, E. F. D. Goufo, and M. Khumalo, "Coexistence of multi-scroll chaotic attractors for fractional systems with exponential law and non-singular kernel," Chaos, Solitons \& Fractals, vol. 139, Article ID 110021, 2020.

[29] H. Jahanshahi, A. Yousefpour, Z. Wei, R. Alcaraz, and S. Bekiros, "A financial hyperchaotic system with coexisting attractors: dynamic investigation, entropy analysis, control and synchronization," Chaos, Solitons \& Fractals, vol. 126, pp. 66-77, 2019.

[30] L. Zhou, C. Wang, X. Zhang et al., "Various attractors, coexisting attractors and antimonotonicity in a simple fourthorder memristive twin-T oscillator," International Journal of Bifurcation and Chaos, vol. 28, no. 4, pp. 481-495, 2018.

[31] B. Bao, L. Xu, N. Wang, H. Bao, Q. Xu, and M. Chen, “Thirdorder RLCM-four-elements-based chaotic circuit and its coexisting bubbles," AEU-International Journal of Electronics and Communications, vol. 94, pp. 26-35, 2018. 
[32] A. Bayani, K. Rajagopal, A. J. M. Khalaf et al., "Dynamical analysis of a new multistable chaotic system with hidden attractor: antimonotonicity, coexisting multiple attractors, and offset boosting," Physics Letters A, vol. 383, no. 13, pp. 1450-1456, 2019.

[33] M. S. Patel, U. Patel, A. Sen et al., "Experimental observation of extreme multistability in an electronic system of two coupled Rössler oscillators," Physical Review E, vol. 89, no. 2, Article ID 022918, 2014.

[34] C. B. Li and J. C. Sprott, "Multistability in the Lorenz system: a broken butterfly," International Journal of Bifurcation \& Chaos, vol. 24, no. 10, p. 7, 2014.

[35] S. Jafari, A. Ahmadi, S. Panahi, and K. Rajagopal, "Extreme multi-stability: when imperfection changes quality," Chaos, Solitons \& Fractals, vol. 108, pp. 182-186, 2018.

[36] B. C. Bao, H. Bao, N. Wang, M. Chen, and Q. Xu, "Hidden extreme multistability in memristive hyperchaotic system," Chaos, Solitons \& Fractals, vol. 94, pp. 102-111, 2017.

[37] S. Zhang, Y. C. Zeng, Z. J. Li et al., "Generating one to fourwing hidden attractors in a novel $4 \mathrm{D}$ no-equilibrium chaotic system with extreme multistability," Chaos, vol. 28, no. 1, Article ID 013113, 2018.

[38] B. A. Mezatio, M. T. Motchongom, B. R. Wafo Tekam, R. Kengne, R. Tchitnga, and A. Fomethe, "A novel memristive 6D hyperchaotic autonomous system with hidden extreme multistability," Chaos, Solitons \& Fractals, vol. 120, pp. 100-115, 2019.

[39] J. P. Singh and B. K. Roy, "Crisis and inverse crisis route to chaos in a new 3-D chaotic system with saddle, saddle foci and stable node foci nature of equilibria," Optik, vol. 127, no. 24, pp. 11982-12002, 2016.

[40] H. Bao, N. Wang, B. C. Bao et al., "Initial condition-dependent dynamics and transient period in memristor-based hypogenetic jerk system with four line equilibria," Communications in Nonlinear Science and Numerical Simulation, vol. 57, pp. 264-275, 2017.

[41] Y. Mukouyama, H. Kawasaki, D. Hara, and S. Nakanishi, "Transient chaotic behavior during simultaneous occurrence of two electrochemical oscillations," Journal of Solid State Electrochemistry, vol. 19, no. 11, pp. 3253-3263, 2015.

[42] S. Sabarathinam, C. K. Volos, and K. Thamilmaran, "Implementation and study of the nonlinear dynamics of a memristor-based duffing oscillator," Nonlinear Dynamics, vol. 87, no. 1, pp. 37-49, 2017.

[43] A. Yousefpour, H. Jahanshahi, J. M. Munoz-Pacheco et al., "A fractional-order hyper-chaotic economic system with transient chaos," Chaos, Solitons \& Fractals, vol. 130, Article ID 109400, 2020. 